\title{
Systems Biology: Science or Technoscience?
}

Karen Kastenhofer

Institute of Technology Assessment, Austrian Academy of Sciences \& Department of Anthropology, University of Vienna

Zitieren als: Kastenhofer, K., 2017, Systems Biology: Science or Technoscience?, in: Green, S. (Hg.): Philosophy of Systems Biology: Perspectives from Scientists and Philosophers: Springer, 157-167.

How and why were you initially drawn to systems biology?

Since finishing my university education in biology, I have been researching epistemic practices and cultures within the life sciences. The experienced fundamental, but unexplained differences between doing ecological field work as opposed to doing molecular biology lab work had left me puzzled and unsatisfied with the very foundations of the scientific discipline I had just earned a degree in. With no fixed curriculum in science and technology studies (STS) offered in Vienna at that time, I underwent a second, selfassembled university education consisting of lectures in philosophy of 
science and STS, courses in qualitative sociological methodology and active participation in a cultural anthropology working group.

Very much in line with this personal source of motivation, my first STS research project ('Science as Culture', starting in 1999) focused on the enculturation of biology students as compared to students of physics, literature and history. The empirical and analytical work performed therein sensitized me for major differences between more or less distant epistemic communities as they are actualized and conveyed via university curricula, including different ontologies, epistemologies, values and habitual norms. This project also helped me understand what it means to belong or not belong to a certain academic tribe in all its multi-facetted day-to-day reality. A second research project ('Cultures of Non-Knowledge', 2005-2007) made me focus on the diverse epistemic cultures ${ }^{1}$ implicit in expert advice on risk regulation (within the fields of agri-biotechnology and mobile phone use) and their roles in the formation and stabilization of expert dissent. Both these projects - 'Science as Culture' and 'Cultures of Non-Knowledge' addressed scientific cultures as a mostly given context of individual action. Even though they left room for an understanding of culture as process and

\footnotetext{
${ }^{1}$ In short, the term 'epistemic cultures' refers to an analysis of science by anthropological means. Scientists are understood as members of a specific research community (or 'tribe'), research is analyzed as a cultural as much as an epistemic practice (for examples of this approach, see Latour, 1987; Becher, 1989; Knorr Cetina 1999).
} 
enactment, something that is again and again re-interpreted and reactualized by individual action, they could build on the assumption that the major ontologies, normative settings and boundary drawings ${ }^{2}$ of a scientific community were not open for dispute; neither processes of enculturation and socialization nor the enactment of expert authority are typical situations in which such fundamental norms would be explicated (at least in the researched cases). Rather, the stability and matter of course character of scientific cultures tend to be over-emphasized in both contexts for didactic as well as strategic reasons.

When Regine Kollek from the University of Hamburg invited me in 2009 to contribute to a research proposal targeting the epistemic culture of systems biology, I was both enthusiastic and cautious. The envisioned project would focus on the question of how systems biology deals with complexity and which specific cultural traits, community characteristics and epistemic practices are developed in this context (going beyond the crude observations that the field is multi-disciplinary, combines 'wet' and 'dry'

\footnotetext{
2 'Boundary drawing' refers to determining who belongs to a given community (and who doesn't), what makes you belong to it (or not belong to it) and how the difference between belonging and not belonging to this community is executed. Traditionally, a given epistemic community (e.g., that of molecular biology) would draw a boundary to other epistemic communities (e.g., that of mathematicians), as well as to other, non-epistemic communities or actor fields (e.g., that of politicians or journalists). For synthetic biology, to give a more recent example, drawing a boundary to 'old' and 'inadequate' attempts at introducing engineering to biology within biotechnology has become an important aspect of identity work. Moreover, boundaries are drawn between 'rightful synthetic biology' or 'synthetic biology in a strict sense' (in one interview called 'the MIT bubble - that is, MIT plus Imperial College") and other approaches that are (allegedly) unrightfully labelled as synthetic biology.
} 
laboratory research in allegedly iterative circles and makes use of mathematical algorithms and computation). It would also provide me with an opportunity to reconstruct the relevance of epistemic cultures within present day-to-day life science research activities (e.g., do systems biologists share specific norms and how do they become relevant in research conduct?). This aspect together with Regine's recognized expertise in both the life sciences and cultural anthropology certainly weighed on the positive side. On the other hand, I was aware of some major challenges of the planned undertaking. (1) Day-to-day-research activity does not highlight cultural stability and boundary drawing as did the two former cases of enculturation and expert dissent. (2) Systems biology allegedly stands for a field in a state of emergence and hence of ongoing changes. (3) It very likely represents a new mode of institutionalizing science, including new modes of field formation, characterization and stabilization. (4) The empirical analysis of systems biology is complicated by systems biology's multi-disciplinary and heterogeneous identity. (5) Understanding the basic scientific aspects of systems biology is tremendously challenging in itself due to its multidisciplinary character, spanning not only cutting edge life science knowledge and methods, but also mathematics and computational science. (6) With systems biology linking local and global realms in particular ways (just as 
every other research field), the decision where to perform the empirical work becomes difficult and influential. And finally (7), in drafting the specific project proposal, it was unclear how a comparative approach that is methodologically prerequisite for the re-construction of epistemic cultures should be set up empirically.

Despite these challenges, the research proposal was successful and I was left with searching for ways to deal with them in a constructive way. In fact, the original research project under the title 'Towards a Holistic Conception of Life? Epistemic Presumptions and Socio-Cultural Implications of Systems Biology' (2010-2013) is now being followed by another project focusing on the '(Techno)Epistemic Cultures of $21^{\text {st }}$ Century Life Science' (2014-2018), directly building on the gathered insights. That leaves me with the opportunity to research systems biology for a time span of more than eight years; a fact that thrills me in a positive way most of the time, but sometimes also makes me despair. Like with most qualitative research, one key to success is to translate the aforementioned challenges into epistemic resources, to see the exclamation marks that are already hidden in the question marks. One major thesis I already gathered from the accumulated material is that systems biology as a (techno)epistemic culture shares a lot of its characteristics with other contemporary Big Science research 
approaches such as modern brain research instead of gaining its identity solely from (a linear advancement of) its historical forerunners. In other words, when looking at its cultural traits, systems biology is firstly characterized as a contemporary Big Science endeavor and only secondly as relating to a molecular biology tradition. When looking at the various subcommunities within systems biology and the interplay of systems and synthetic biology, the picture of course becomes more complex. These observations also raise the question what kind of category 'systems biology' represents: a discipline, an inter-discipline, a sub-discipline, a research approach or a new paradigm?

How do you view the relation between philosophy and systems biology, and (how) can these fields inform each other?

Speaking from an ethnography of science point of view, It seems to be one of the peculiarities of systems biology that philosophical questions have been raised since its establishment (that is, around the year 2000) in central scientific fora and that scientists as well as philosophers of science actively contributed to their discussion. The volume of this early philosophical reflection adds to, but also goes beyond the more general trend to 
accompany the kick-off-phase of newly emerging fields with a rather high percentage of review articles as compared to research papers. In close proximity to the emergence of systems biology, we can observe a rekindling of some old(er) philosophical questions of the life sciences: discourses on vitalist versus mechanistic ontologies, on reductionism versus holism, on Lamarckism vs. Darwinism; conceptual discussions about evolution (that is, 'natural', 'artificial' and 'instrumental' evolution), complexity, emergence and autopoiesis; methodological debates about the integration of qualitative and quantitative, epistemic and technological approaches.

With the active and frequent involvement of philosophy/philosophers, systems biology has been attributed with a nimbus that makes us think of $19^{\text {th }}$ century conceptions of science, when science and philosophy were much closer to each other and science - then labelled as "philosophy of nature" or "Naturgeschichte" - was subsumed under the general category of philosophy. Such an anachronistic re-make of the field's habitus is also facilitated by systems biology's inter- or transdisciplinary ambition (the latter assignment following Stichweh's definition of transdisciplinarity, see Stichweh, 1994) and systems biologist's attempts to overcome the high degree of disciplinary differentiation and segregation that was institutionalized over the past century. A further observation (that has yet to 
be validated methodologically) adds to this line of thought: systems biology papers tend to quote earlier literature than other comparable scientific papers. Points of reference that date back to the midst of the $20^{\text {th }}$ century are no uncommon features of such publications.

Other, more practical aspects of systems biology also stimulate an active exchange between philosophy and systems biology: systems biology as an inter- or transdisciplinary undertaking is already trained in addressing the burdens of multi-disciplinary collaboration and communication, hence facilitating an exchange with philosophy as yet another field of expertise. Moreover, the attempt alone to address the experienced difficulties and ambiguities of interdisciplinarity leads systems biologists into philosophical territory. And, last but not least, systems biology is also a project of emancipation from traditional (mostly molecular biology) approaches that can only benefit from addressing specificities from a third point of view. A rekindling of debates on what life is and how it can be grasped scientifically is potential tailwind for its legitimization.

On the other hand, current systems biology clearly is a $21^{\text {st }}$ century undertaking. That means that broadly speaking, philosophical debates are rated as a "nice to have" add on, not as a sine qua non of day-to-day research practice. Much of doing systems biology does not necessitate any explicit 
philosophical reflection and we are far from a situation where philosophers of science are indispensable members of any systems biology project or where philosophy of science represents an obligatory part of any academic career in systems biology. There are no traces to be found of an implicit hierarchy that would subsume science inter alia under a broader philosophical ambition. Rather, it seems to depend on the individual systems biologist's mindset whether and how far philosophical considerations become influential in scientific practice (and, like in most current science, philosophers are not deemed prerequisite by biologists for "getting philosophical"). If a broader ambition of science is addressed (explicitly or implicitly), then it tends to point towards overarching engineering paradigms rather than philosophical ones, towards changing worlds and constructing new worlds rather than understanding them.

What do you consider the most neglected topics and/or contributions in late $20^{\text {th }}$ Century (philosophy of) biology?

As outlined above, my own approach is not a strictly philosophical one. Although much of what I do may fall within the realm of a "philosophy of scientific action" or epistemology, other aspects of my work could count as 
sociology of science or laboratory ethnography. To answer this question in a fruitful way, I will therefore have to widen the scope to all meta-reflection on science as practice, community and/or culture.

The one strand of analysis I have most often been searching for in vain or with unsatisfactory results, consists of historical analyses of the development and re-definition of biology as a discipline from its early beginnings until now. ${ }^{3}$ A short and superficial glance at the curricula of the University of Vienna reveals distinct changes in the categorization of biology - from its early beginnings as a "natural history" subsumed initially within medical education and then under the broader category of "philosophy", until its emancipation as a discipline within the canon of natural sciences and finally its recent differentiation into biology and molecular biology, both taught within the faculty of the "life sciences". What were the struggles accompanying these processes of re-labelling? What triggered the underlying changes in ontology? What did they answer to? What relevance did they have for situated epistemologies and epistemic practices? What is the current reality and state of the category "biology" as seen against this historical background?

\footnotetext{
${ }^{3}$ Obviously, detailled case studies on specific biological specialties, research institutions and historical phases abound (see for example Nyhart 1995 or Strasser 2002). Biology's place and identity in (a) general epistemic landscape(s) as mirrored by its specific placings in academic institutions has on the other hand retained the character of an empirical anecdote rather than leading to a socio-epistemological debate.
} 
Due to many understandable reasons, historians/philosophers of science have become rather cautious about any longue durée statements and only a few scholars dare make the jump from micro-histories to macro history or macro histories (here, I think of theorists of science and technology like Dominique Pestre, John Pickstone, Andrew Jamison or Steven Shapin). As a consequence, there is no broader scholarly debate about the major changes biology underwent and their scientific as well as societal relevance. This scholarly silence stands in stark contrast to existing more exoteric discourses (mostly situated in the contexts of science policy and science communication) that strongly draw on talk about radical shifts and disruptive developments - one only has to think of the alleged convergence of sciences and technologies, the power attributed to a new generation of technosciences ${ }^{4}$ or the claim that synthetic biology converts biology into precision engineering.

A second strand that would be highly fruitful for my work is closely related to this first one: it comprises the area where philosophies of science, philosophies of technology and philosophies of engineering overlap. Again, there is some scholarly literature that bridges this existing gap (for example work by Davis Baird, Jean-Pierre Dupuy, Andrew Feenberg, Don Ihde, Alfred

\footnotetext{
${ }^{4}$ For an outline and illustration of the concept of technoscience, see Nordmann (2006).
} 
Nordmann or Jutta Weber), but it still represents an exception rather than the mainstream I would favor it to be. I also emphasize the plural of philosophies here, because local differences in the conceptions and implementations of science, technology and engineering persist until today, in defiance of all globalization and standardization efforts, and they become highly relevant in the modes in which science, technology and engineering are amalgamated in newly emerging research fields. Synthetic biologists would for instance speak about an "MIT and Imperial College bubble" when it comes to characterizing the state of the art of their field, referring to an engineering paradigm that is (allegedly) specific to the Massachusetts Institute of Technology. Other sources stress the specific cultural background of Japanese research that would blur the boundary between nature and artifacts or science and technology (cp. Steeghs, 2011) and thereby possibly also such distinctions like the one between systems and synthetic biology.

A third strand of questions that should attract more interest in my view comprises the epistemological ramifications of Big Science (cp. Vermeulen et al., 2010), including its high degree of collaborativity, multi-sitedness, digitalization, (alleged) orientation towards external goals and/or orientation towards multiple goals and its high reliance on singular, pre-defined projects. Classical epistemological conceptions of science mostly invoke the idea of 
an individual thinker who over time gains insights or methodologically supported convictions, following a specific research paradigm, relating to a specific scientific community (most prominently, Ludwik Flecks initial analyses, see Fleck, 1979). With Big Science projects, thinking and thinkers or even individual observers belonging to something like a thought collective seem radically outdated. Research is conceived of as a happening with multiple aims and multiple outputs that accrue at multiple places if only the overall architecture of a project works well enough. Building a scientific career equals surviving being part of as many of such processes as possible without losing too much epistemic credibility while gathering a maximum of citation points. It can be read as a new philosophical stand-point as well as a mirror of the development that current epistemological analyses in STS favor focusing on actor-networks rather than individual thinkers. But whether the development and success of Latour and Callon's Actor-Network-Theory should be read and applied not only as the former, but also as the latter, is still open for debate.

What have been the most significant advances in systems biology? 
Systems biology has been set up as a new research approach, even as a new paradigm of doing research in a specific scientific domain, that is, broadly speaking, modern molecular biology with its high throughput technologies and resulting Big Data. In the wake of preparing for and aiming towards realizing this new approach, the engaged scientists developed new concepts, methods and last but not least new insights and product lines. But what I see in the center of systems biology's achievements is really the acknowledgement of an existing epistemic shortcoming in recent molecular biology and the ensuing attempt to reframe fundamental aspects of doing research in this domain in implicit as well as explicit terms so as to answer to these shortcomings. One could critically assess that not in all cases the epistemic struggle was the first priority and that other stakes were involved in the launch of systems biology - in short, that systems biology was simply a new buzzword and a new strategy to sell more of the same science. Such an interpretation could be tied to relatively meager results when it comes to the fulfillment of initial claims of systems biology's proponents to help solve almost every current societal problem, from hunger to climate change, and it may not be completely unsubstantiated. But still, it does not seem to cover all efforts undertaken by all actors in this context. 
Individual researchers and whole research groups took a high risk in investing in an approach that is not guaranteed to "deliver", that necessitates high individual and collective investments (building up new research networks, engaging in multidisciplinary collaboration, acquiring new expertise, questioning well established practices and hierarchies and changing existing curricula) and lures natural scientists out from a rather cozy position of not having to reflect upon the broader epistemic ramifications and ontological presumptions of what they do in their day-to-day work. At least some biologists (as well as philosophers, mathematicians, etc.) sincerely bought into this new project and achieved a lot in re-discussing and re-constructing what it means to do research in the current life sciences and which promising options have not yet been considered adequately. It may rather be a side effect that the attempt to tame Big Data in molecular biology also led to re-addressing the big questions of biology itself, at least punctually: what is specific to the living world as compared to the non-living and what is specific to a science of the living world as compared to a science focusing on the non-living? How does biology address complexity, diversity and evolvability? What is the relation between (the represented) nature, a computer model and something we build brick by brick? How can "wet" and "dry" research be integrated in a meaningful way (and why do we have to 
integrate them at all)? How can the organization of doing science be adapted to a changing conception of living organisms and/or of how living organisms can be researched?

Hence, from my point of view, the most significant advances made in the context of systems biology are related to a certain audacity to re-frame existing questions and ask new ones - new questions that do not lend themselves to being easily answered (or easily published in the research section of high ranking scientific journals). It may not be the next in silico model of a cell, that will be a major achievement of systems biology, but the questions that are raised and discussed while aiming at building such a model.

What do you consider the most important problems in (philosophy of) systems biology and what are the prospects for progress in this respect?

Problems that face those doing systems biology research can be divided into two groups: problems that relate to the general situation of current (techno)science, of doing (techno)science and being in (techno)science on the one hand and problems that are specific to systems biology on the other. 
In the following, I will start each topic with an outline of the general context before focusing in on those aspects specific to systems biology.

(1) In relation to the more general context, one grand question is: what is or should be the identity of science - i.e., what are its primary, defining orientations along which individual actions can be positioned and quality can be assessed? This first question underwent periods of explicit debate, of historical change and relative stabilization; it is closely related to the characterization of the relation between science and society or science and other societal subsystems. Currently, debates and practical struggles that allude to this theme are very lively in various contexts (such as science funding, quality assessment and science regulation), but tend to address the underlying grand question only implicitly. In rare cases individual stakeholders address it directly, such as in opinions about the current contract between science and society. In other cases, such as the discourse on the character of synthetic biology, specific sub-themes are spotlighted, like the double-identity of research as science as well as engineering or a shift from science towards engineering. But most of these cases fall short of including all available cases and options and/or do not open up a debate on all relating factors and consequences. The prominent science plus/versus engineering discourse in synthetic biology would necessitate an in-depth 
analysis of what it means to orient (individual and collective) action primarily towards understanding or construction or creation and what the consequences of an amalgamation of orientations in hybrid understanding/engineering activities are (Kastenhofer, 2013; the incompatibility of scientific and technological purposes is also mentioned in Xavier et al. 2014, 488).

(2) Another grand question related to the more general context is raised by major changes in the organization and practice of science: how is it possible to perform science under the current organizational and practical conditions (project based research, technology and data driven research, collaborative networks, multi-disciplinary and multi-center approaches, etc.) and what kind of science results from it? Even if science is clearly and primarily oriented towards gaining new insights, how do we conceive of this process in a Big Science context? And where does it leave the individual mind, the individual scientist, that feature so centrally in traditional epistemological thought? Of course, there are epistemological approaches that attempt to capture this situation, both within philosophy of science and in science itself. Systems biologists developed their own microscale answers, like the scheme of iterative circles, connecting exploratory data analysis, hypothesis-testing laboratory experiments and in silico model 
construction. But it might also be time for a more disruptive re-framing of what science is, how knowledge is produced and what it means to know something.

One of the central problems systems biology has chosen to address is to make sense of high quantities of data about very small entities of living systems with the help of mathematics and computer modelling. In addressing this problem, specific techniques, collaborative patterns and digital constructs gained interest in their own right. Moreover, building a computer model that would enable us to simulate nature in a reliable way, to replace nature in epistemic processes and eventually enable the replacement of nature in processes of material production, somehow emerged as a fascinating vision and objective, with a power to mobilize people and resources far beyond the realm of the life sciences. Again, in the three-step formulation of the central problem addressed by systems biology, we find inscribed a gradient from systems biology being a science, to systems biology representing a technoscience or a technoscientific vision. For the first incarnation of systems biology, a lack of available robust and appropriate data might represent a real bottle neck. Also, and this has been mentioned many times, the integration of different existing approaches (be 
they called top-down, bottom-up and middle-out or more traditional physiology and quantitative analysis or else) poses a major challenge.

(3) A third grand problem relates to securing quality standards in doing science. Given current situations and contexts of doing science, I want to stress one specific aspect: as science has become ever more multidisciplinary, multi-sited and collaborative and focuses increasingly on scientific as well technological and societal issues, (successful) communication has become an ever more challenging and crucial component of doing (good) science. Much of this challenge is being addressed in very creative ways, like building up new networks (e.g. in the context of developing new research agendas and funding initiatives), introducing new types of infrastructures (e.g. multi-disciplinary centers, data infrastructures, etc.), experimenting with new formats of education and socialization (e.g. iGEM, the International Genetically Engineered Machine Competition, cp. Bensaude Vincent 2013) or stressing new norms (e.g. "responsible research and innovation" at EU level). Where I see less activity and a growing potential for quality loss is the realm of language and within this realm the assessment and maintenance of (a) terminological accuracy and (b) soundness in formulating goals and visions. The story of the multiple definitions, usages and understandings of terms like "gene" in 
diverse research fields may be old hat, but it addresses an unsolved and increasingly crucial problem. The same holds true for critique concerning empty promises frequently formulated in science communication. One might hold that both of these problems are confined to the popularization of science and do not interfere with core science activities. But with a shift of where "core science" takes place in a multi- and trans-disciplinary paradigm of doing science, the related problems cannot be shook off that easily. What do formulations like "modeling a cell" or "whole-cell modelling" mean? What criteria must be fulfilled in order to be allowed to speak of having "modelled a cell" or having built a "whole-cell model"? What level of performance is realistic in replacing "material results of an evolution in context" like living cells by material, technologically constructed objects like minimal cells or by digital objects like computer models in epistemic and/or technological terms? What are the diverse meanings of terms such as 'system', 'complexity' or 'reductionist' and 'holistic'? And, on a much more general level, what kind of contribution to solving grand societal problems can we expect from technoscientific undertakings and how is this contribution to be assessed? Admittedly, some of these questions are being addressed in single systems biology review articles and contributions from the philosophy of systems biology, at least the terminological ones (for examples see for instance 
Xavier et al. 2014 for 'minimal cells' or Krohs and Callebaut, 2007, for 'models of everything'), but I have found little evidence for a broader uptake of these discussions. I believe that much of the progress in the field of systems biology will depend on such reflection and specification work.

Acknowledgement: This contribution is based on the project ,(Techno)epistemic cultures in 21 st century life sciences' funded by the Austrian Science Fund (FWF) as project number V-383 (Elise Richter position), Oct. 2014 to January 2019.

References

Becher, T. (1989). Academic Tribes and Territories. Intellectual enquiry and the cultures of disciplines. Milton Keynes, Bristol: SRHE\&Open University Press.

Bensaude-Vincent, B. (2013). Discipline-building in synthetic biology. Studies in History and Philosophy of Biological and Biomedical Sciences, 44, 122-129. 
Fleck, L. (1979). The Genesis and Development of a Scientific Fact, (edited by T.J. Trenn and R.K. Merton, foreword by Thomas Kuhn). Chicago: University of Chicago Press.

Kastenhofer, K. (2013). Two sides of the same coin? The (techno)epistemic cultures of systems and synthetic biology. Studies in History and Philosophy of Biological and Biomedical Sciences, 44, 130-140.

Knorr Cetina, K. (1999). Epistemic cultures: how the sciences make knowledge. Cambridge, MA: Harvard University Press.

Krohs, U. and Callebaut, W. (2007). Data without models merging with models without data, in: Boogerd, F. C., Bruggeman, F. J., Hofmeyr, J.-H. S. \& Westerhoff, H. V. (Eds): Systems Biology. Philosophical Foundations (pp. 181-213), Amsterdam: Elsevier.

Latour, B. (1987). Science in action. How to follow scientists and engineers through society. Cambridge, MA: Harvard University Press.

Nordmann, A. (2006). Collapse of Distance: Epistemic Strategies of Science and Technoscience, Danish Yearbook of Philosophy, 41, 7-34. 
Nyhart, L. K. (1995). Biology Takes Form: Animal Morphology and the German Universities, 1800-1900. Chicago: University of Chicago Press.

Steeghs, V. (2011). Nanotechnology \& TA in Japan. Den Haagh: Rathenau Institute.

Stichweh, R. (1994). Wissenschaft, Universität, Professionen. Soziologische Analysen, Frankfurt/Main: Suhrkamp Taschenbuch.

Strasser, B. J. (2002). Institutionalizing molecular biology in post-war Europe: a comparative study. Studies in History and Philosophy of Biological and Biomedical Sciences, 33, 533-564.

Vermeulen, N., Parker, J. N. \& Penders, B. (2010). Big, small or mezzo? EMBO reports, 11, 420-423.

Xavier, J. C., Patil, K. R. \& Rochaa, I. (2014). Systems Biology Perspectives on Minimal and Simpler Cells, Microbiology and Molecular Biology Reviews, 78(3), 487-509. 